\title{
SIMULAÇÃO DA DISTRIBUIÇÃO ESPACIAL DA ÁGUA EM IRRIGAÇÃO LOCALIZADA SUBSUPERFICIAL
}

\author{
Maria Vilma Tavares de Moura ${ }^{1}$, Sérgio Marques Júnior ${ }^{2}$ \\ e Paulo Rodolfo Leopoldo ${ }^{3}$
}

\begin{abstract}
RESUMO
O presente trabalho teve como finalidade o estudo da dinâmica da água no solo, através da irrigação localizada subsuperficial, utilizando-se procedimentos de simulação numérica, com o desenvolvimento de um modelo iterativo computacional, procurando-se estimar, com base em dados de características de retenção de água no solo (teor de água inicial, parâmetros da curva de retenção da água no solo e condutividade hidráulica saturada) e características do emissor (vazão) a distribuição do teor de água no meio após determinado intervalo de tempo. Para validação do modelo foram realizados ensaios em condição de campo em um solo classificado como Terra Roxa Estruturada, "intergrade" para Latossolo Vermelho-Escuro, textura média/argilosa. Foram realizados 4 ensaios com vazões de 5,6 $6 \mathrm{~h}^{-1} \mathrm{e}$ $3,3 \ell \cdot \mathrm{h}^{-1}$, quando utilizados um emissor, e vazões de $4,3 \ell \cdot \mathrm{h}^{-1} \mathrm{e} 2,2 \ell \cdot \mathrm{h}^{-1}$, e dois emissores, funcionando simultaneamente. Utilizando-se o Teste t para comparação de médias, verificou-se uma concordância aceitável entre valores amostrados e simulados. Foi realizada, ainda, uma análise de erro do processo de simulação, através de um coeficiente de desvio espacial (CDE) representado pela relação entre o teor de água volumétrico simulado e o teor de água volumétrico amostrado. Os valores encontrados para esses desvios variaram na ordem de $-14 \%$ a $+18 \%$, que podem ser considerados aceitáveis viabilizando, assim, a utilização do modelo.
\end{abstract}

Palavras-chave: água, simulação, irrigação localizada subsuperficial

\section{SIMULATION OF SPACIAL WATER DISTRIBUTION IN SUBSURFACE DRIP IRRIGATION}

\begin{abstract}
The objective of this work was to study the dynamics of water movement in soils under subsurface drip irrigation, using numeric simulation procedures with the development of a interactive computational model. The main objective was to estimate, using soil-water retention parameters (initial soil-water content, soil-water retention curve and saturated hydraulic conductivity) and emitter parameters (emitter flow), the spatial distribution of moisture in soil after a time increment. To calibrate the model, tests were made under field conditions in a Ultisol "intergrade" to typic Oxisol (texturel medium/clay). Four tests were performed under a flow of $5.6 \ell \cdot \mathrm{h}^{-1}$ and $3.3 \ell \cdot \mathrm{h}^{-1}$ to a single emitter, and a flow of $4.3 \ell \cdot \mathrm{h}^{-1}$ and $2.2 \ell \cdot \mathrm{h}^{-1}$ when two emitters worked simultaneously. Simulated and observed data showed good agreement when the Student's t-Test for mean comparision was used. Also performed was a simulation error analysis (spatial deviation coefficient), expressed as a relation between the simulated and observed soil-water content. Results showed that deviations ranged from $-14 \%$ to $+18 \%$.
\end{abstract}

Key words: water, simulation, subsurface drip irrigation

\footnotetext{
${ }^{1}$ Dr. Secretaria da Agricultura e Abastecimento do Estado do Rio Grande do Norte, Br 101, km 0, Lagoa Nova, fone: (084)2311212, CEP 57075-050, Natal-RN

2 Prof. MSc. Departamento de Engenharia Rural - CCA/UFSC, Rodovia Admar Gonzaga, km 3, Itacorubi, CEP 88037-500, Florianópolis, SC, fone: (048)234-1013. E-mail: smarques@mbox1.ufsc.br

3 Prof. Titular., FCA/UNESP - Departamento Engenharia Rural, fone: (014) 8213883, fax: (014)821-3438, CEP 18603-970, Botucatu, SP
} 


\section{INTRODUÇÃO}

A agricultura irrigada requer o desenvolvimento ou o aperfeiçoamento de métodos e práticas que visem aumentar a eficiência de utilização do recurso hídrico para a produção das culturas. Esta preocupação vem sendo demonstrada claramente nas últimas décadas, nas quais a irrigação localizada tem-se destacado como um dos métodos mais eficientes para aplicação de água em cultivos intensivos, principalmente nas plantações de árvores frutíferas. Tem sido identificado, também, como o sistema que apresenta os melhores índices de relação benefício-custo no processo de produção.

Em relação ao dimensionamento das estruturas hidráulicas para fornecimento de água, é passível de concordância que este deveria ser realizado visando à maximização da produtividade, o que só é possível quando do conhecimento das relações hídricas que a cultura estabelece com o meio, conhecimento este que pode ser utilizado a partir de dados de potencial e teor de água no solo.

A preocupação em se determinar essa característica física do solo dentro da engenharia de irrigação, vem sendo demonstrada pela maioria dos pesquisadores da área, independente do método de irrigação a ser utilizado; entretanto, na irrigação localizada o conhecimento da capacidade de armazenamento de água no solo pode ser, de certa forma, relegado a um segundo plano, já que este é um método onde se procura fornecer uma lâmina de irrigação aproximadamente igual à demanda hídrica da cultura, com alta frequiência.

Mais complexo se torna o estudo desse sistema quando se utiliza a irrigação localizada subsuperficial, onde a água é distribuída sob a superfície do solo, impossibilitando a visualização da frente de molhamento e, em consequiência, uma estimativa da área molhada.

Este impasse mostra a importância do estabelecimento de critérios para se projetar um sistema de irrigação localizada subsuperficial, em que o estabelecimento do espaçamento entre emissores é de extrema importância e uma alternativa seria se conhecer a distribuição espacial da água no solo, com o objetivo de delimitar a região com o teor de água adequado ao desenvolvimento das plantas. Para a viabilidade dessas análises, podem ser utilizados processos de simulação matemática dos eventos físicos, haja vista o grande número de variáveis atuantes no meio, como aquelas empregadas nos trabalhos de Botrel (1988) e Freitas (1996).

Com base nas argumentações até aqui discutidas, o presente trabalho tem como objetivo, a partir de procedimentos de simulação numérica, o estudo da distribuição espacial da água no solo em irrigação localizada subsuperficial, procurando estabelecer, com base unicamente em dados de características de retenção de água no solo e características hidráulicas do emissor, subsídios para a estimativa do espaçamento de emissores e a configuração da distribuição de água no meio após determinado intervalo de tempo.

\section{DESENVOLVIMENTO TEÓRICO DO MODELO}

O modelo de simulação proposto baseia-se no trabalho de Botrel (1988) e visa estimar o movimento de água entre pontos adjacentes no solo, cujo fluxo de água é função do gradiente de potencial hidráulico total entre esses pontos. Imaginando-se que o solo pode ser representado por uma seqüência tridimensional de células hipotéticas adjacentes, é possível pressupor-se que o movimento da água pode se dar em 3 direções, definidas pelas coordenadas mecânicas x, y e z, conforme apresentado na Figura 1. Neste contexto, procurou-se empregar métodos numéricos, tomando-se como base a técnica de diferenças finitas. $\mathrm{O}$ movimento de água que ocorre no solo, partindo da entrada de água no sistema através do emissor em um ponto subterrâneo, foi estimado dentro de intervalos de tempo predeterminados, em compartimentos do solo. Este modelo atua de forma tridimensional, estimando o teor de água no solo, após um intervalo de tempo "Dt" de funcionamento para um emissor, ou mesmo dois emissores, funcionando simultaneamente, espaçados de uma distância "l".

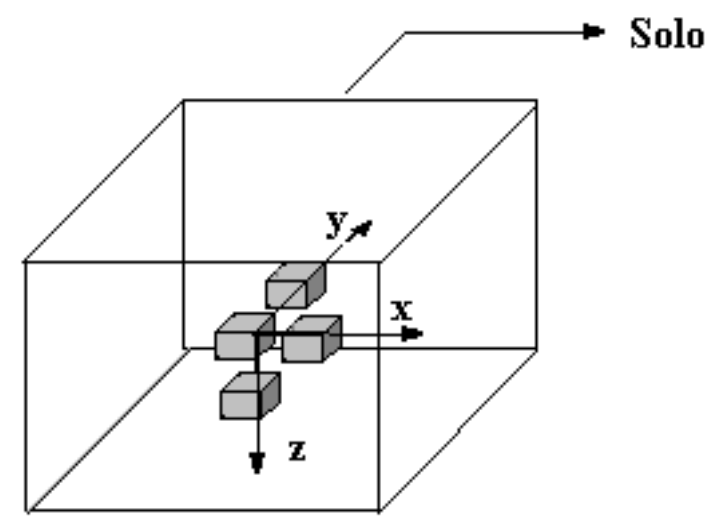

Figura 1. Configuração hipotética de camadas de solo

Objetivando-se quantificar a variação do teor de água que ocorre em cada camada de solo ao longo do tempo, o sistema em questão foi considerado isotérmico e incompressível, cujas funções potencial mátrico e condutividade hidráulica em meio não saturado, são conhecidas para cada célula imaginária.

Nessa idealização hipotética, a quantidade de água (em termos de fluxo "q") capaz de se transferir de uma célula a outra, pode ser estimada a partir da equação de Buckingham-Darcy (Swartzendruber, 1969) que, adaptada para o modelo, assume a forma

$$
\overrightarrow{\mathrm{q}}_{\mathrm{i} \leftrightarrow \mathrm{i}+1}=-\mathrm{K}(\boldsymbol{\theta})_{\mathrm{i} \rightarrow \mathrm{i}+1} \cdot\left(\frac{\mathrm{H}_{\mathrm{i}+1}-\mathrm{H}_{\mathrm{i}}}{\mathrm{dx}}\right)
$$

$\mathrm{e}$

$$
\overrightarrow{\mathrm{q}}_{\mathrm{j} \leftrightarrow \mathrm{j}+1}=-\mathrm{K}(\boldsymbol{\theta})_{\mathrm{j} \rightarrow \mathrm{j}+1} \cdot\left(\frac{\mathrm{H}_{\mathrm{j}+1}-\mathrm{H}_{\mathrm{j}}}{\mathrm{dy}}\right)
$$

e

$$
\overrightarrow{\mathrm{q}}_{\mathrm{k} \leftrightarrow \mathrm{k}+1}=-\mathrm{K}(\theta)_{\mathrm{k} \rightarrow \mathrm{k}+1} \cdot\left(\frac{\mathrm{H}_{\mathrm{k}+1}-\mathrm{H}_{\mathrm{k}}}{\mathrm{dz}}\right)
$$

em que $\overrightarrow{\mathrm{q}}_{\mathrm{i} \leftrightarrow \mathrm{i}+1}$ corresponde ao modelo de fluxo da água da posição i da célula hipotética para a posição i+1 da célula hipotética adjacente, ou vice-versa $\left|\mathrm{L}^{\mathrm{T}} \mathrm{T}^{-1}\right|$, $\mathrm{K}(\theta)_{\mathrm{i} \rightarrow \mathrm{i}+1}$ corresponde ao valor da condutividade hidráulica entre as células hipotéticas localizadas nas posições i e a sua adjacente $\mathrm{i}+1\left|\mathrm{~L} . \mathrm{T}^{-1}\right|, \mathrm{H}_{\mathrm{i}}$ e $\mathrm{H}_{\mathrm{i}+1}$ equivalem, respectivamente, ao potencial hidráulico total na posição i da célula hipotética e posição i+1 da célula hipotética adjacente $|\mathrm{L}|$ e $\mathrm{dx}$ a distância horizontal entre o centro das células hipotéticas localizadas nas posições i e i+1. Essa mesma conformação é apresentada nas equações (2) e (3) para as posições hipotéticas j e k. 
O valor correspondente à função condutividade hidráulica $(K(\theta))$ em um tempo " $t$ ", que nesse estudo é estimado através do modelo de Van Genuchten (1980) pode ser obtido como sendo a média aritmética dos valores encontrados nas células imaginárias, ou seja:

$$
\mathrm{K}(\theta)_{\mathrm{i} \rightarrow \mathrm{i}+1}=\left(\frac{\mathrm{K}(\theta)_{\mathrm{i}}+\mathrm{K}(\theta)_{\mathrm{i}+1}}{2}\right)
$$

e

$$
\mathrm{K}(\theta)_{\mathrm{j} \rightarrow \mathrm{j}+1}=\left(\frac{\mathrm{K}(\boldsymbol{\theta})_{\mathrm{j}}+\mathrm{K}(\boldsymbol{\theta})_{\mathrm{j}+1}}{2}\right)
$$

$\mathrm{e}$

$$
\mathrm{K}(\theta)_{\mathrm{k} \rightarrow \mathrm{k}+1}=\left(\frac{\mathrm{K}(\theta)_{\mathrm{k}}+\mathrm{K}(\theta)_{\mathrm{k}+1}}{2}\right)
$$

Por definição, a densidade de fluxo da água corresponde a um volume de água que passa por uma unidade de área e por unidade de tempo; portanto, o incremento de umidade volumétrica que vai ocorrendo nas células hipotéticas ao longo do tempo em função do movimento da água no solo, pode ser estimado como sendo:

$$
\Delta \theta_{i, j, k}=\left(q_{i x} \cdot A_{i x}+q_{j y} \cdot A_{j y}+q_{k z} \cdot A_{k z}\right) \cdot \frac{\Delta t}{V_{T}}
$$

em que $\Delta \theta_{i, j, z}$ corresponde ao incremento de umidade volumétrica que sofre a célula localizada na posição i, j e z, $\left|\mathrm{L}^{3} \cdot \mathrm{L}^{-3}\right|, \mathrm{q}_{\mathrm{ix}}, \mathrm{q}_{\mathrm{jy}}$ e $\mathrm{q}_{\mathrm{kz}}$ representam os fluxos nas posições $\mathrm{i}, \mathrm{j}, \mathrm{k}$ nas direções x, y e $\mathrm{z}$, respectivamente, $\mathrm{A}_{\mathrm{ix}}, \mathrm{A}_{\mathrm{jy}}$ e $\mathrm{A}_{\mathrm{kz}}$ representam a seção transversal ao fluxo nas direções x, y e z, respectivamente $\left|\mathrm{L}^{2}\right|, \Delta \mathrm{t}$ equivale ao intervalo de tempo considerado para a quantificação do movimento de água dentro do processo iterativo que se estabelece, $|\mathrm{T}|$, e $\mathrm{V}_{\mathrm{T}}$ corresponde ao volume total considerado da célula hipotética $\left|\mathrm{L}^{3}\right|$.

O incremento do teor de água na base de volume que ocorre em uma célula hipotética, durante um intervalo de tempo, promove alteração no valor do gradiente de potencial hidráulico total entre células adjacentes gerando, então, um processo iterativo de movimentação e distribuição de água no solo. Neste modelo, a redistribuição de água começa a partir da célula cuja posição corresponde à localização do emissor, posição em que o incremento de água que se dá ao longo do tempo é função do acréscimo de água durante o período no qual ocorre a irrigação, podendo ser definido como:

$$
\Delta \theta_{\text {EMISSOR }}=\frac{\mathrm{Q} \cdot \Delta \mathrm{t}}{\mathrm{V}_{\mathrm{T}}}
$$

em que Q corresponde à vazão do emissor $\left|\mathrm{L}^{3} \mathrm{~T}^{-1}\right|$.

\section{Ensaios de campo}

Os ensaios foram conduzidos em uma área experimental do Departamento de Engenharia Rural, no Campus de Botucatu, da Faculdade de Ciências Agronômicas, pertencente à Universidade Estadual Paulista "Júlio de Mesquita"- UNESP/FCA, localizada no município de Botucatu, Estado de São Paulo. O solo do local foi classificado por Carvalho et al. (1983) como Terra Roxa Estruturada "intergrade" para Latosssolo Vermelho-Escuro, distrófico, textura média/argilosa, cujos resultados da análise granulométrica são apresentados na Tabela 1 .
Tabela 1. Análise granulométrica e classe textural do solo estudado em função da profundidade

\begin{tabular}{ccccc}
\hline $\begin{array}{c}\text { Profundidade } \\
(\mathrm{m})\end{array}$ & \multicolumn{2}{c}{ Análise Granulométrica $\left(\mathrm{g} \cdot \mathrm{kg}^{-1}\right)$} & Classe \\
\cline { 2 - 4 } & Areia & Silte & Argila & Textural \\
\hline $0-0,20$ & 523,8 & 110,4 & 365,8 & Argilosa \\
$0,20-0,40$ & 540,4 & 102,3 & 357,3 & Argilosa \\
$0,40-0,60$ & 533,8 & 93,8 & 372,4 & Argilosa \\
$0,60-0,80$ & 554,2 & 80,0 & 365,8 & Argilosa \\
$0,80-1,00$ & 509,2 & 91,8 & 399,0 & Argilosa \\
\hline
\end{tabular}

Com o objetivo de aferir o modelo proposto para os ensaios de campo, foram coletadas amostras indeformadas, utilizando-se anéis amostradores de 0,044m de diâmetro e 0,046m de altura, utilizados para determinação da curva de retenção de água no solo, massa específica do solo e condutividade hidráulica em meio saturado, com três repetições para cada profundidade e parâmetro determinado. Visando obter melhor representação, foram coletadas amostras representativas das camadas 0-0,20; 0,20-0,40; 0,40-0,60; 0,60-0,80 e 0,80-1,00m.

Para a estimativa da curva de retenção de água, as amostras coletadas foram previamente saturadas em laboratório e submetidas ao processo de secagem, com tensões que variaram de 0,00 a aproximadamente $1500,00 \mathrm{kPa}$. Utilizou-se o método da mesa de tensão para tensões de 0,00 até aproximadamente 7 kpa e, acima deste valor, foi utilizada a câmara de pressão de Richards. Os valores obtidos para o teor de água volumétrico $(\theta)$ e potencial matricial $(h)$ para cada amostra nas diversas profundidades, foram ajustados ao modelo proposto por Van Genuchten (1980) através de regressão não linear, cujo valor do teor de água volumétrico é dado pela expressão:

$$
\theta=\theta_{\mathrm{r}}+\frac{\theta_{s}-\theta_{\mathrm{r}}}{\left[1+(\alpha \cdot|\mathrm{h}|)^{n}\right]^{m}}
$$

sendo que " $\theta$ " diz respeito ao teor de água com base em volume atual do solo $\left|\mathrm{L}^{3} . \mathrm{L}^{-3}\right|$, " $\mathrm{q}_{\mathrm{r}}$ " corresponde ao teor de água residual (neste estudo será considerado como sendo o teor de água encontrado ao potencial matricial correspondente a $-1500 \mathrm{kPa}$ ) e " $\theta_{s}$ " refere-se ao teor de água no ponto de saturação (teor de água a $0 \mathrm{kPa}$ de tensão no solo). Estas funções têm a dimensão $\left|L^{3} \cdot L^{-3}\right|$. Os coeficientes " $\alpha$ “, " $n$ " e "m" são relacionados ao solo e "h" ao potencial matricial |L|.

$\mathrm{O}$ procedimento iterativo foi codificado em um algoritmo expresso em linguagem Pascal no ambiente Turbo, sendo realizado durante o tempo total ao qual se queira inferir sobre a condição da distribuição do teor de água no solo.

Os resultados obtidos na simulação foram definidos para um perfil de solo com $1 \mathrm{~m}$ de profundidade (plano vertical) subdividido em camadas de $0,20 \mathrm{~m}$. Quando considerado um emissor em funcionamento, a distância horizontal simulada foi de $1 \mathrm{~m}$, cujas amostragens foram subdivididas em cinco pontos distantes de $0,20 \mathrm{~m}$, totalizando 25 pontos amostrados (plano horizontal); quando considerados dois emissores, o perfil foi subdividido em quatro pontos distantes de $0,20 \mathrm{~m}$, também divididos em cinco camadas de $0,20 \mathrm{~m}$ de profundidade, totalizando 20 pontos de amostragem.

Os valores do teor de água observados foram obtidos através dos ensaios realizados no campo, a fim de aferir o modelo de simulação, para as mesmas distâncias e profundidades já mencionadas para o processo de simulação em todos os ensaios.

Os ensaios I e II compreendem os testes em que foi utilizado um emissor, cujas vazões foram de $5,6 \ell \cdot \mathrm{h}^{-1}$ e $3,3 \ell \cdot \mathrm{h}^{-1}$, respectivamente, tendo sido amostrado o perfil do solo 24 e 48 
horas após o início de aplicação de água; os ensaios III e IV, com vazões de $4,3 \ell . h^{-1}$ e $2,2 \ell . h^{-1}$, compreendem os testes em que foram utilizados dois emissores funcionando simultaneamente. Neste caso, os resultados foram obtidos somente 24 horas após o início de aplicação de água, uma vez que os pontos coletados se encontravam entre os emissores inviabilizando, assim, uma segunda amostragem no local. Em todos os testes, o tempo de aplicação de água foi de 4 horas. Para validação dos resultados dos teores de água no solo simulados e observados, foram utilizados três procedimentos, entre os quais foi aplicado um método de análise estatística, utilizando-se o Teste " $t$ " de Student, para determinar a que nível de probabilidade os dois conjuntos de amostra (simuladas e observadas) provenientes de duas populações, possuem a mesma média. Utilizou-se, também, o coeficiente de correlação de Pearson, que reflete a extensão de uma relação linear entre os dois conjuntos de dados.

De posse dos dados do teor de água no solo, e decorrido um intervalo de tempo dt após o início do processo de irrigação, procurou-se compará-los com os dados simulados obtidos através do processo iterativo idealizado, cujos resultados são discutidos a seguir.

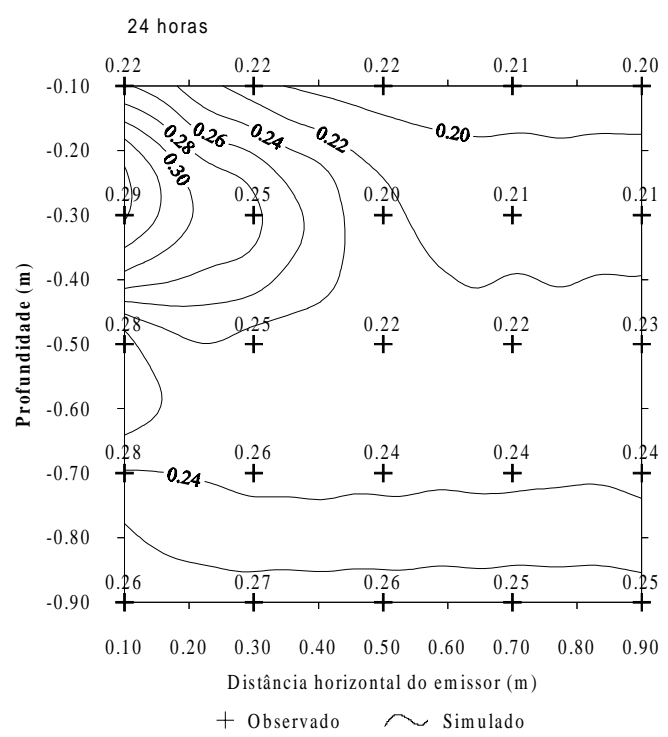

\section{RESULTADOS E DISCUSSÃO}

Os valores simulados foram obtidos através do modelo computacional proposto, com base nos parâmetros condutividade hidráulica em meio saturado, dados da curva de retenção de água no solo, massa específica do solo e teor de água inicial, além de algumas características do emissor referentes à vazão e profundidade de instalação.

O incremento de tempo utilizado no processo de simulação (Dt) foi de $10 \mathrm{~s}$, totalizando o período de 24 e 48 horas, quando utilizado um emissor apenas, e de 24 horas quando utilizados dois emissores funcionando simultaneamente, espaçados regularmente de $1 \mathrm{~m}$, tempo este contado a partir do início da entrada de água no sistema através de emissor(es) localizado(s), em todos os casos, a $0,25 \mathrm{~m}$ sob a superfície do solo.

Para melhor visualização dos resultados do processo de simulação e do comportamento da distribuição de água nos ensaios de campo, as Figuras 2 a 4 apresentam a distribuição de água no perfil do solo simulado e observado, a partir de linhas que representam o mesmo teor de água no solo e pontos amostrados, respectivamente.

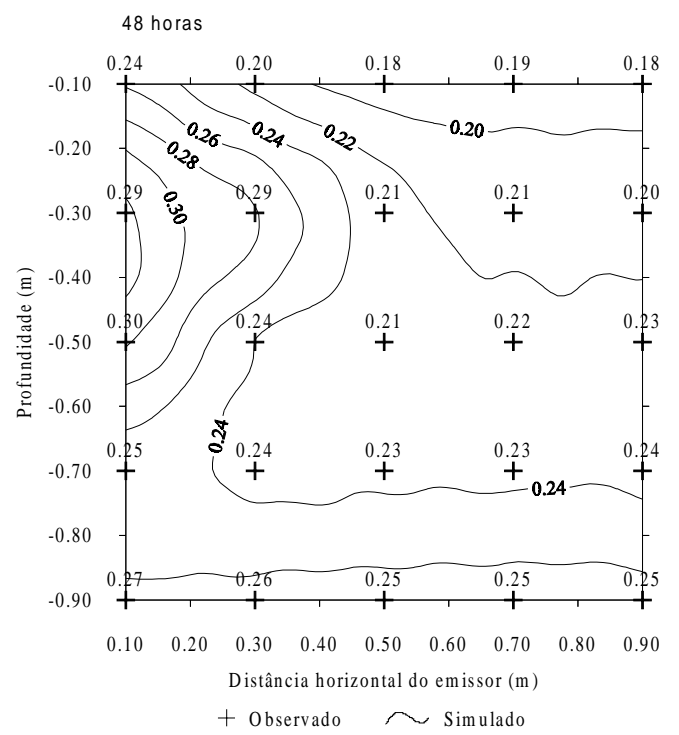

Figura 2. Valores simulados e observados do teor volumétrico de água no solo, referentes ao Ensaio I, com vazão de 5,6 $\ell . h^{-1}, 24 h$ e 48h após o início da aplicação de água
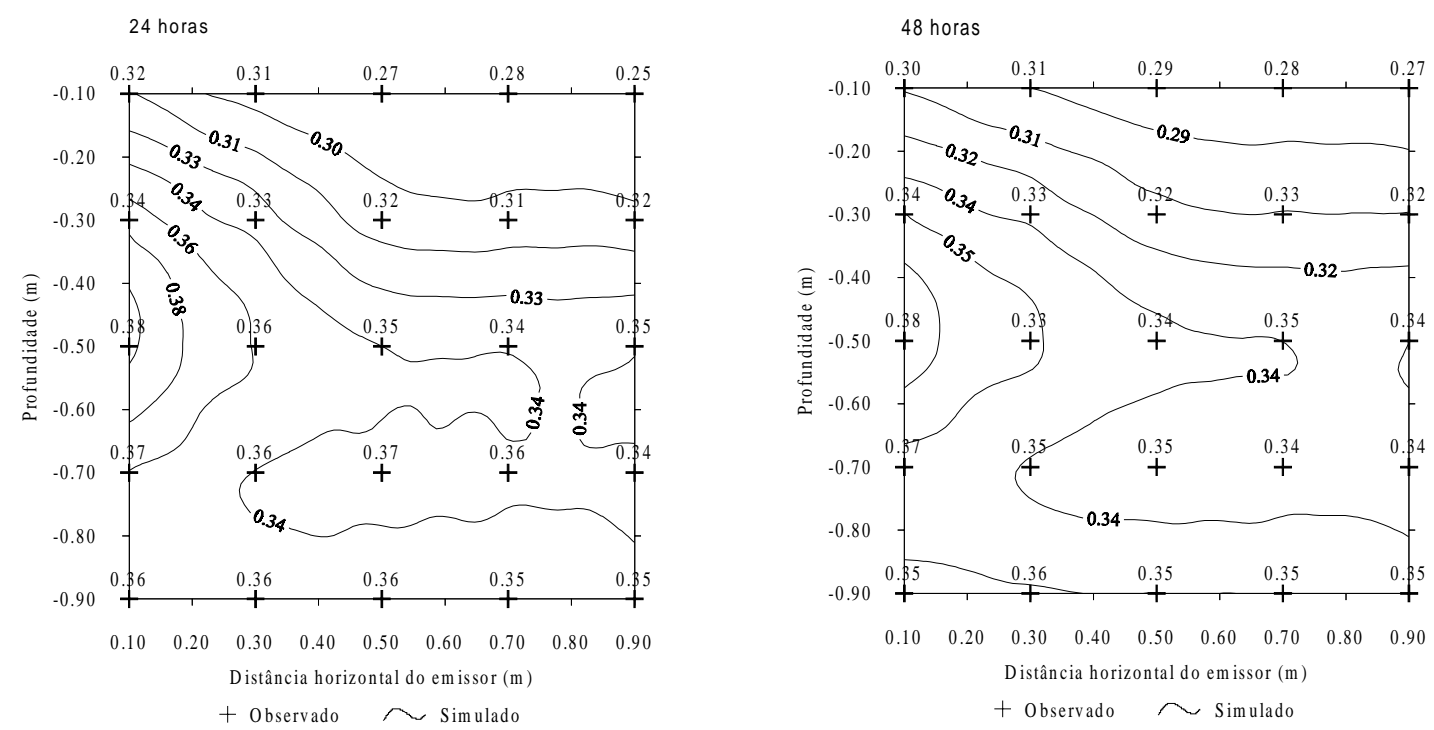

Figura 3. Valores simulados e observados do teor volumétrico de água no solo, referentes ao Ensaio II, com vazão de 3,3 .h ${ }^{-1}, 24 h$ e 48 h após o início da aplicação de água 

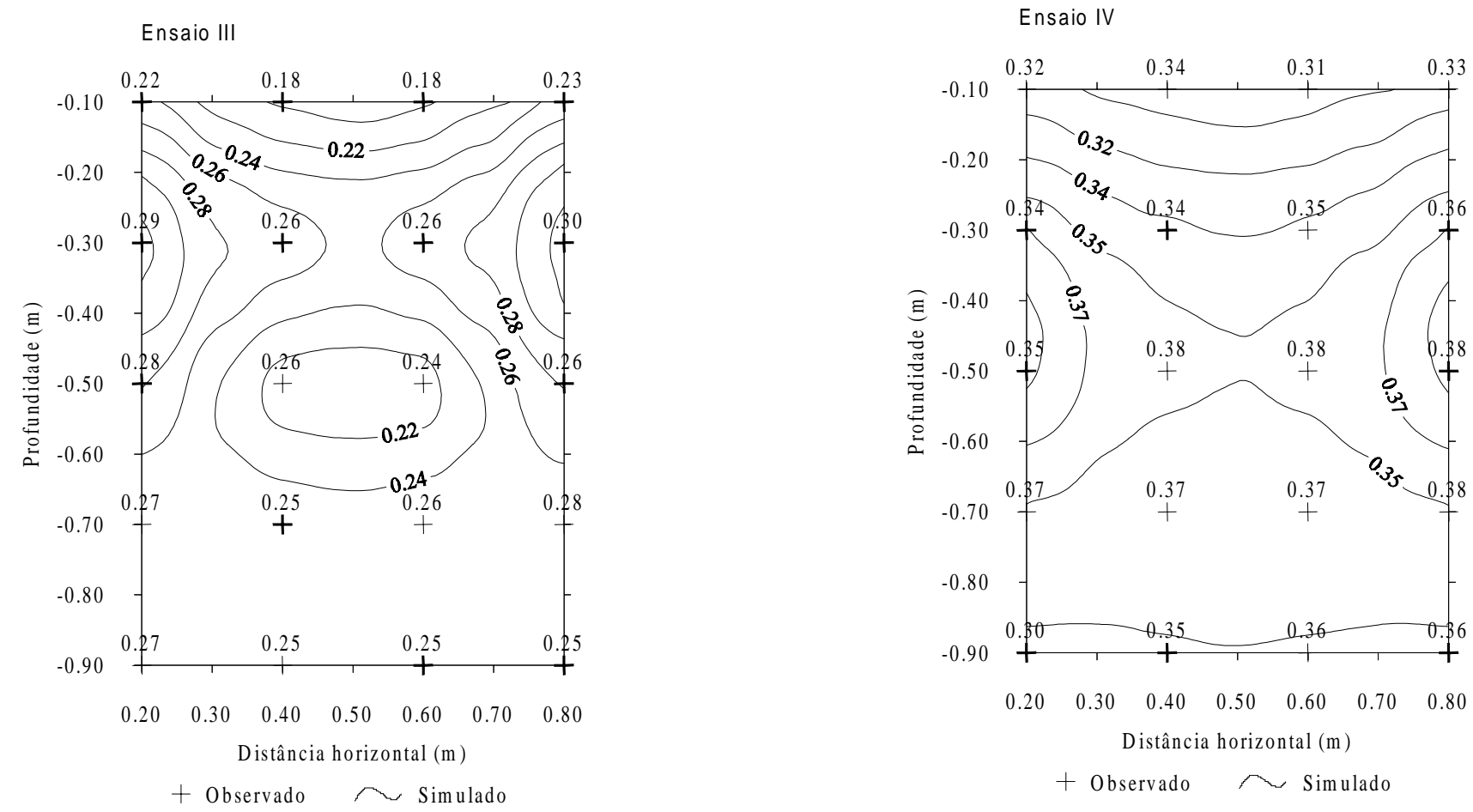

Figura 4. Valores simulados e observados do teor volumétrico de água no solo, referentes ao Ensaio III, com vazão de 4,3 $\ell \cdot \mathrm{h}^{-1}$, e Ensaio IV, com vazão de 2,2 $\ell \cdot \mathrm{h}^{-1}, 24 \mathrm{~h}$ após o início da aplicação de água

O resultado da análise estatística é apresentado na Tabela 2.

Tabela 2. Resultados da análise estatística para valores médios do teor de água no solo $\left(\mathrm{m}^{3} \cdot \mathrm{m}^{-3}\right)$ simulados e observados

\begin{tabular}{lcccc}
\hline Ensaio & \multicolumn{2}{c}{ Valores médios $\left(\mathrm{m}^{3} \mathrm{~m}^{-3}\right)$} & Teste t & Correlação de Pearson \\
\cline { 2 - 4 } & Simulados & Amostrados & & \\
\hline $\mathrm{I}(24 \mathrm{~h})$ & 0,2420 & 0,2396 & 0,7905 & 0,8175 \\
$\mathrm{I}(48 \mathrm{~h})$ & 0,2414 & 0,2352 & 0,5212 & 0,9472 \\
$\mathrm{II}(24 \mathrm{~h})$ & 0,3359 & 0,3360 & 0,9938 & 0,9086 \\
$\mathrm{II}(48 \mathrm{~h})$ & 0,3332 & 0,3343 & 0,8780 & 0,8780 \\
$\mathrm{III}(24 \mathrm{~h})$ & 0,2560 & 0,2530 & 0,7670 & 0,8210 \\
$\mathrm{IV}(24 \mathrm{~h})$ & 0,3510 & 0,3510 & 0,9150 & 0,4590 \\
\hline
\end{tabular}

Analisando-se a tabela acima, é possível observar-se, através do Teste $t$, que existe a probabilidade, que varia de $76,70 \%$ a $99,38 \%$, de que as médias pertençam a um mesmo conjunto de dados, com exceção do Ensaio I (48h) que apresentou resultados com apenas $52,12 \%$ de probabilidade de que as médias simuladas e observadas pertençam a um mesmo conjunto de dados, resultado este considerado insatisfatório; no entanto, é importante destacar que tanto o processo de simulação quanto os resultados observados são altamente influenciados pelas características dos parâmetros físicos medidos principalmente por sua variabilidade espaço-temporal, o que implica, por certo, em condições de anisotropia, não previstas para utilização no modelo de simulação. Em relação ao coeficiente de correlação de Pearson, verifica-se alta correlação linear entre os valores simulados e amostrados, com exceção do ensaio IV, que apresentou coeficiente de correlação de 0,495 , apesar do valor do Teste $t$ apresentar a probabilidade de $91,50 \%$ de que as médias façam parte de um mesmo conjunto de dados.

A análise estatística empregada refere-se a um estudo sobre a disposição geral do processo de simulação, e o Teste t à média das populações; entretanto, mais importante que a comparação das médias, é a verificação de como se distribui este erro entre o teor de água simulado e o observado, ao longo do perfil do solo. Assim sendo, torna-se relevante analisar a distribuição espaço-temporal do erro gerado durante o processo de simulação, cujas considerações são feitas a seguir.

\section{Análise de erro no processo de simulação}

Neste estudo, a análise do erro no processo de simulação foi efetuada a partir da relação entre os resultados simulados e os observados em cada ponto amostrado no solo. Convencionouse chamar esta relação de coeficiente de desvio espacial (CDE) representado da seguinte forma:

$$
\mathrm{CDE}=\frac{\theta_{\mathrm{SIM}}}{\theta_{\mathrm{OBS}}}
$$

em que $\theta_{\text {SIM }}$ corresponde ao teor de água volumétrico simulado e $\theta_{\mathrm{OBS}}$ ao teor de água volumétrico observado em cada posição do solo. Através desta relação, é possível estimar-se a super ou subestimativa que ocorre em cada posição do solo, quando da utilização do modelo de simulação. Inicialmente e para melhor visualização do fenômeno, as Figuras 5 e 6 apresentam a representação gráfica desses desvios.

É possível observar que no ensaio I, com o tempo de amostragem de 24 horas (Figura 5) o coeficiente de desvio espacial mostra, de forma geral, que ocorre superestimativa dos valores do teor de água no solo através do processo de simulação nas regiões mais próximas do emissor e, também da mesma forma, subestimativa dos valores de teor de água em posições mais distantes da fonte pontual. Esta mesma tendência apresenta-se no ensaio II, análise realizada no tempo de amostragem referente a 24 horas.

Com relação à amostragem realizada 48 horas após o início do processo de irrigação, verifica-se que esta tendência não é tão nítida, já que o erro (tanto a superestimativa quanto a subestimativa do modelo de simulação) é distribuído mais 
24 horas

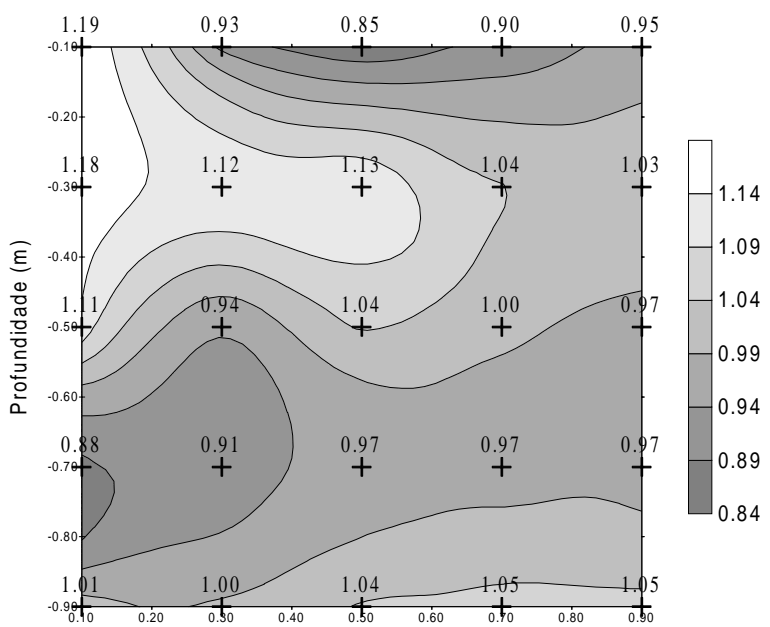

Distância horizontal do emissor $(\mathrm{m})$
48 horas

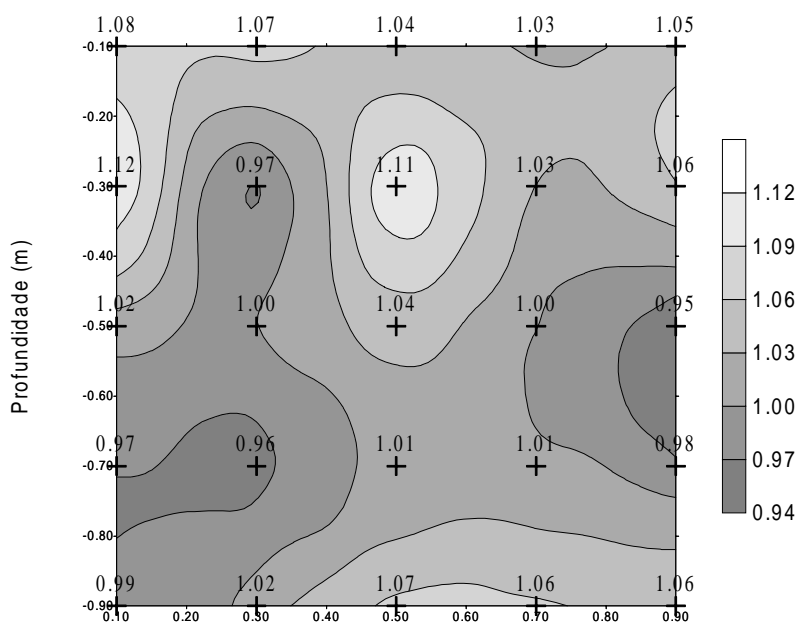

Distância horizontal do emissor $(m)$

Figura 5. Coeficiente de desvio espacial referente ao Ensaio I, 24 e 48 horas após o início de aplicação de água
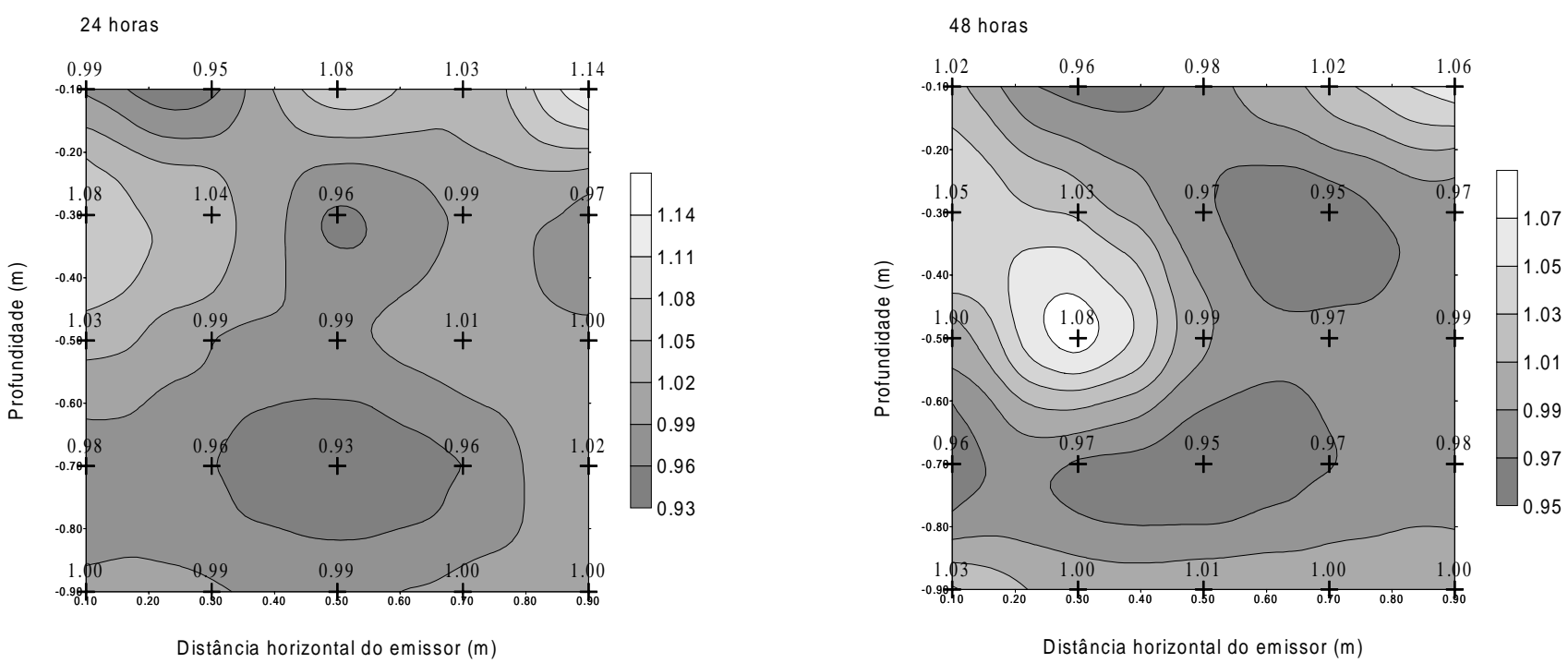

Figura 6. Coeficiente de desvio espacial referente ao Ensaio II, 24 e 48 horas após o início de aplicação de água

distante da fonte pontual, principalmente no teste apresentado no ensaio II (Figura 6). Observa-se, ainda, que o erro de estimativa, aqui denominado coeficiente de desvio espacial, é menor nas avaliações realizadas após 48 horas que nas realizadas após 24 horas do início do processo de irrigação. Esses desvios podem estar relacionados a problemas de histerese e mesmo à utilização de um procedimento de cálculo da média, utilizado pelo teste estatístico.

Neste aspecto, diversos trabalhos citam que o modelo de estimativa da função condutividade hidráulica apresentado por Van Genuchten, subestima o valor desse parâmetro (Alexander \& Skaggs,1986, Bacchi, 1988) principalmente nas regiões cujo teor de água se encontra próximo à saturação, que é o caso dos pontos próximos ao emissor. Este fato pode ser o responsável pelas considerações referentes à superestimativa, já que uma subestimativa da condutividade hidráulica simula uma movimentação menor de água no perfil e, conseqüentemente, concentração de água em regiões perto da fonte pontual. Outros modelos de simulação de movimentação de água no perfil do solo, que também se utilizam dos procedimentos apresentados por Van Genuchten (Marques Júnior,1992, Freitas, 1996) apresentaram efeitos similares de super e subestimativa entre perfis simulados e amostrados do teor de água no solo, evidenciando a importância desta questão.

A Figura 7 apresenta o coeficiente de desvio espacial para os ensaios realizados com dois emissores espaçados regularmente de $1 \mathrm{~m}$, sendo possível, também, verificar a superestimativa que ocorre junto ao emissor e a subestimativa nos pontos mais distantes.

Os resultados apresentados pela Figura 7 são aqueles que devem apresentar o maior interesse, já que se referem à simulação do teor de água de um perfil de solo localizado entre dois emissores enterrados, que pode ser adaptada como alternativa, por exemplo, para o procedimento de cálculo do espaçamento de emissores em irrigação localizada subsuperficial. Em ambos os casos encontraram-se desvios da ordem de 14 e $18 \%$ para a superestimativa e de 10 a $18 \%$ para a subestimativa, que podem ser considerados plenamente aceitáveis, uma vez que, como já comentado, os dados de entrada do modelo que se referem aos parâmetros do solo (condutividade hidráulica em meio saturado, curva de retenção de água e teor de água inicial), sofrem intensa variabilidade espaço-temporal, o que dificulta a caracterização de tais parâmetros em determinado tipo de solo; contudo, estudos sobre a variabilidade espacial de solos nas últimas décadas têm despertado especial atenção entre os pesquisadores, com o surgimento de novas 
Ensaio III

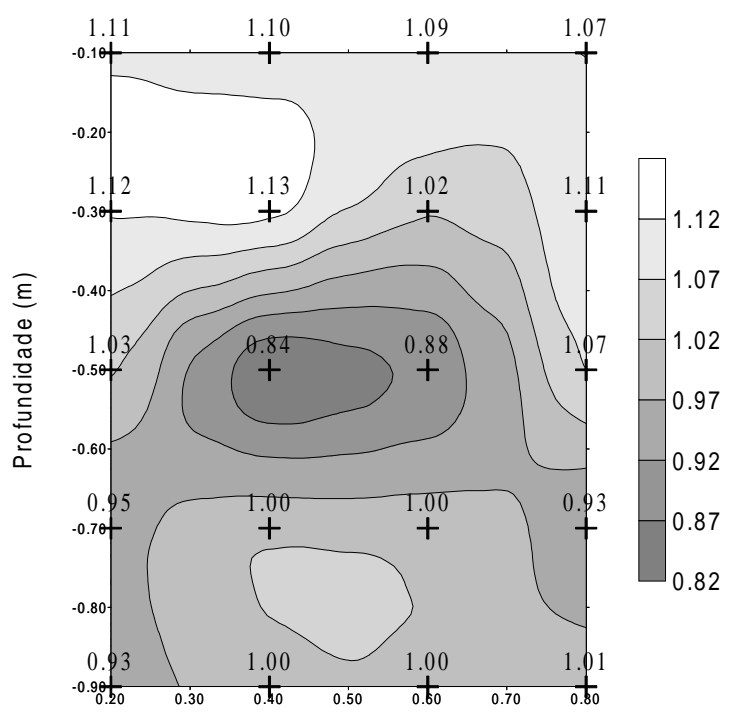

Distânicia horizontal do emissor $(m)$

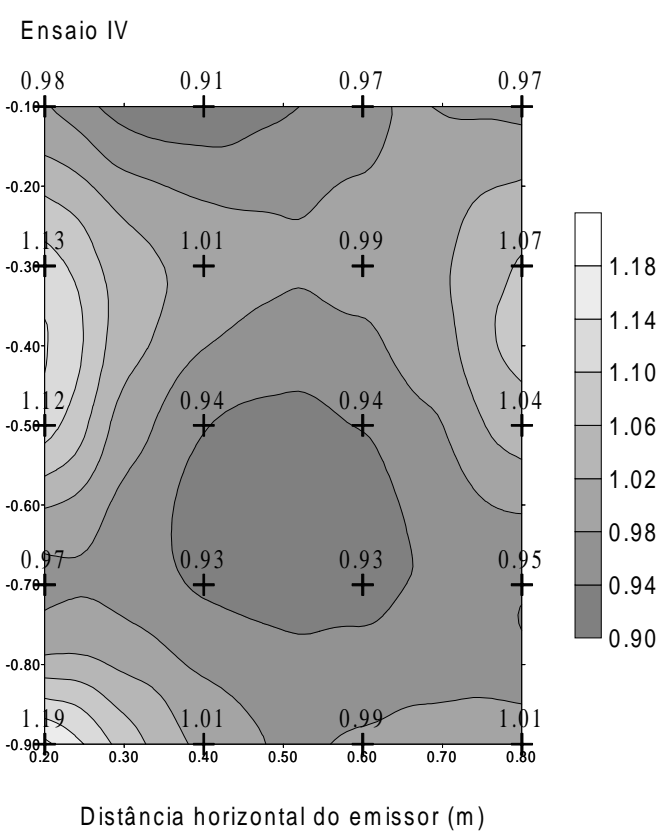

Figura 7. Coeficiente de desvio espacial referente aos Ensaios III e IV, 24 horas após o início de aplicação de água

ferramentas para a análise deste problema, uma vez que os métodos estatísticos considerados clássicos, não tratam o problema de forma explícita. A introdução de métodos de análise baseados na teoria das variáveis regionalizadas utilizadas inicialmente por Nielsen, da Universidade da Califórnia, EUA, como comentam Reichardt et al. (1986) possibilitou a verificação de que muitas propriedades do solo obedecem a uma tendência de distribuição normal, mas outras, como a condutividade hidráulica do solo, seguem distribuições assimétricas.

No caso do teor de água no solo, Vachaud et al. (1985) apresentam um estudo da estabilidade temporal da variabilidade espacial das medidas de umidade, mostrando que determinados pontos de observação do teor de água no solo sempre apresentam um desvio mínimo do valor médio do campo experimental. Os resultados obtidos neste trabalho confirmam esta tendência, não justificando, assim, uma análise sobre o parâmetro em questão.

Quanto à curva de retenção de água no solo, é passível de concordância que erros inerentes ao processo de histerese, podem ocorrer, mas a complexidade desse processo dificulta sua descrição quantitativa, tendo sido desconsiderados os seus efeitos no modelo de simulação.

\section{CONCLUSÕES}

Com base nas discussões referentes aos resultados apresentados neste trabalho, pode-se concluir que:

1. Nas condições em que o trabalho foi conduzido, o modelo de simulação proposto aproxima os valores do teor de água no solo, quando comparados com valores obtidos em ensaios de campo.

2. Variações na ordem de $-14 \%$ e $+18 \%$ entre valores simulados e amostrados, podem ser consideradas aceitáveis, devido ao fato de que as variáveis de entrada do modelo se referem, essencialmente, a parâmetros físicos do solo, sujeitos a intensa variabilidade espaço-temporal.

\section{REFERÊNCIAS BIBLIOGRÁFICAS}

ALEXANDER, L.; SKAGGS, R.W. Predicting unsaturated hydraulic conductivity from the soil water characteristic. Transactions American Society of Agricultural Engineers,. v.29, p.176-84, 1986.

BACCHI, O.O.S. Teoria dos fatores de escala na análise comparativa de métodos de comparação da condutividade hidráulica de um solo. Piracicaba: ESALQ, 1988. 94 p. Tese Doutorado.

BOTREL, T.A. Simulação da distribuição espacial da água em solo irrigado com gotejador. Piracicaba: ESALQ, 1988. 61p. Tese Doutorado.

CARVALHO, W.A.; ESPÍNDOLA, C.R.; PACCOLA, A.A. Levantamento de Solos da Fazenda Lageado, Estação Experimental "Presidente Médici". Boletim Científico Faculdade Ciências Agronômicas, UNESP (Botucatu), n.1, p.1-95, 1983.

FREITAS, E.P. Simulação bidimensional da dinâmica da água em um sulco de irrigação. Piracicaba: ESALQ, 1996. 109p. Tese Doutorado.

MARQUES JUNIOR, S. Modelo computacional para simular a equação da velocidade de infiltração da água no solo. Piracicaba: ESALQ, 1992. 83p. Dissertação Mestrado.

REICHARDT, K.; VIEIRA, S.R.; LIBARDI, P.L. Variabilidade espacial de solos e experimentação de campo. Revista Brasileira Ciência do Solo, v.10, p.1-6, 1986.

SWARTZENDRUBER, D. Soil-water behavior as described by transport coefficients and functions. Advances in Agronomy, v.18, p.327-63, 1969.

VACHAUD G.; PASSERAT, D.; SILANS, A.; BALABANIS, P.; VAUCLIN, M. Temporal stability of spatially measured soil water probability density function. Soil Science Society American Journal, v.49, p.822-8, 1985.

VAN GENUCHTEN, M. Th. A closed form equation for predicting the hydraulic conductivity of unsatured soils. Soil Science Society of America Journal, v.44, p.892-8, 1980. 